\title{
Multilanguage e-Learning Course for Industrial Automation
}

\author{
Jaromír Hrad, Tomáš Zeman and Jiř́i Hájek \\ Czech Technical University in Prague, Faculty of Electrical Engineering, \\ Department of Telecommunication Engineering \\ Technická 2, CZ-166 27 Praha 6, Czech Republic \\ \{hrad,zeman,hajekj3\}@fel.cvut.cz, http://www.comtel.cz
}

\section{Summary}

Compared to traditional forms of education, we can find many advantages for increasing the qualification with the help of interactive e-learning courses. Let us mention for example lower training costs, almost unlimited capacities (concerning the number of students taking the course simultaneously) and the possibility to return (even repeatedly) to already studied topics. The e-learning methods also bring opportunity to choose the most appropriate pace of training.

The Leonardo da Vinci project ELefANTS (E-Learning for Acquiring New Types of Skills) that was concluded in the autumn 2007 had the main objective to help various groups of people (also the handicapped ones) to enter the labor market and increase their opportunities to be integrated into the work process.

The main contribution of the project consists in the development and evaluation of e-learning tools - two independent courses - that support the placement of people from the target groups in the job market. The first course, within its nine modules, provides an overview of working with a personal computer from the very beginning, explaining the fundamental concepts of networking, teleworking and Customer Relationship Management (CRM); the second one introduces the contemporary automation technology and its relation to teleinformatics. Let us briefly introduce the latter.

Besides the topic, the most important detail is that the entire course is available in 7 different languages (English, French, German, Czech, Slovak, Polish and Slovenian), which (together with the multilingual multimedia dictionary that is also available online) dramatically increases its usability and opens a new dimension that helps to overcome barriers in the united Europe; the trainees get access to a reference material containing large volume of special terminology, facilitating not only their professional growth, but also the improvement of their language skills. 
The course can be visited online at "http://elefants.cvut.cz/", currently still free of charge. The necessary equipment of the prospective users is as simple as a common personal computer with Internet browser (and network connection, of course). The visitor can register for the course and choose a login name and password. After receiving the confirmation of registration by e-mail the study may begin.

The course is divided into the following modules covering the areas of industrial automation and teleinformatics: Automation, its Scope and Importance; Control Systems; Digital Telecommunication Networks, Radio and Satellite Communications, GSM, NGN; Fundamentals of Design Using PLC; Logical Systems; Digital Systems, Controllers and Filters; Simulation and Modelling of Real Processes; Rudiments of Fuzzy Logic Summary; Artificial Intelligence in Automation; Reliability in Technical Systems; Recent Trends and Expectations in Control Systems. The thematic relations among the individual modules are relatively loose, which means that no specific order of study is required.

We have also prepared a project for further development of the courses. Specifically, we plan to increase the number of available languages, emphasizing the European dimension, and to employ more interactive elements, such as animations, simulations and self-evaluation tests. We hope that such changes will greatly improve the usability and attractiveness of the products for their final recipients. The results should be available in the autumn 2009 . 